\title{
A novel MTTT mutation m.15933G > A revealed in analysis of mitochondrial DNA in patients with suspected mitochondrial disease
}

Heidi K. Soini ${ }^{1,2,3,4^{*}}$, Antti Väisänen ${ }^{1,2,3}$, Mikko Kärppä ${ }^{1,2,3}$, Reetta Hinttala ${ }^{2,4,5}$, Laura Kytövuori ${ }^{1,2,3}$, Jukka S. Moilanen ${ }^{2,6,7}$, Johanna Uusimaa ${ }^{2,4,5}$ and Kari Majamaa ${ }^{1,2,3}$

\begin{abstract}
Background: Mitochondrial diseases present with variable multi-organ symptoms. Common disease-causing mutations in mitochondrial DNA (mtDNA) are regularly screened in diagnostic work-up, but novel mutations may remain unnoticed.

Methods: Patients $(N=66)$ with a clinical suspicion of mitochondrial disease were screened for their mtDNA coding region using conformation sensitive gel electrophoresis and sequencing. Long-PCR was used to detect deletions followed by POLG1 sequencing in patients with multiple deletions.

Results: We discovered three novel mtDNA variants that included m.8743G > C, m.11322A > G and m.15933G > A. The novel MTTT variant m.15933G > A is suggested to be pathogenic. Analysis revealed also multiple mtDNA deletions in two patients and five nonsynonymous variants that were putatively pathogenic according to in-silico prediction algorithms. In addition, a rare haplogroup $\mathrm{H}$ associated m.7585_7586insT variant was discovered.

Conclusions: Among patients with a suspected mitochondrial disease, a novel MTTT variant m.15933G > A was discovered and is suggested to be pathogenic. In addition, several putatively pathogenic nonsynonymous variants and rare variants were found. These findings highlight the importance of coding region mtDNA screening among patients with clinical features suggesting a mitochondrial disease, but who lack the common mitochondrial disease mutations.
\end{abstract}

Keywords: mtDNA, Mitochondrial disease, MTTT, Insertion, Multiple deletions, POLG1

\section{Background}

More than 200 pathogenic point mutations and largescale rearrangements have been described in mitochondrial DNA (mtDNA) [1]. Some of the most common mitochondrial diseases caused by mutations in mtDNA are Kearns-Sayre syndrome, Leber's hereditary optic neuropathy, myoclonic epilepsy with ragged red fibers syndrome, MELAS syndrome (mitochondrial encephalomyopathy, lactic acidosis with stroke-like episodes) and

\footnotetext{
* Correspondence: heidi.soini@oulu.fi

${ }^{1}$ Research Unit of Clinical Neuroscience, Neurology, University of Oulu, P.O. Box 5000FI-90014 Oulu, Finland

${ }^{2}$ Medical Research Center Oulu, Oulu University Hospital and University of Oulu, P.O. Box 5000FI-90014 Oulu, Finland

Full list of author information is available at the end of the article
}

the syndrome of neuropathy, ataxia and retinitis pigmentosa (NARP). The prevalence of mitochondrial diseases caused by mtDNA mutations has been calculated to be 1:5000 in the adult population $[1,2]$ and the total prevalence rises up to 1:4300, when disorders caused by mutations in nuclear-encoded genes contributing to mitochondrial functions are included [3].

Screening of established pathogenic mtDNA and nDNA mutations is straightforward, but detection of novel or rare mtDNA mutations is more tedious. Such mutations may remain unnoticed, unless the entire mtDNA coding region is sequenced after excluding known mitochondrial disease mutations. However, conventional sequencing methods do not detect large mtDNA deletions, nor are 
they good in recognizing extremes of mtDNA heteroplasmy. It is also imperative to be able to analyse the pathogenic potential of novel or rare mtDNA mutations and to determine their frequency in the population [4], as mtDNA is highly variable by nature. There is also emerging evidence suggesting that polymorphic variation can influence disease phenotype and raise the risk for developing a mitochondrial disorder [5-9]. Furthermore, mtDNA variants have been suggested to increase the risk of common neurodegenerative diseases, such as Alzheimer's and Parkinson's disease [10-13]. In this sense, the Finnish population is ideal for detecting underlying genetic risk factors, as it is considered to be a genetically isolated, homogenous population.

In the present study, we screened the mtDNA coding region of 66 patients with a clinical suspicion of a mitochondrial disease. Our aim was to determine mtDNA sequence for rare mtDNA variants and to assess if they were pathogenic mutations or polymorphic risk factors. Conformation sensitive gel electrophoresis (CSGE) and sequencing were used to determine the mtDNA coding region of the patients and long-PCR was used to detect multiple mtDNA deletions. Polymerase $\gamma$ gene (POLG1) was sequenced in multiple deletions patients.

\section{Methods}

\section{Patients and population controls}

Finnish patients $(N=66)$ with a clinically suspected mitochondrial disease were examined. Muscle samples $(N=21)$, blood samples $(N=36)$ or both $(N=9)$ were obtained. Common symptoms among selected patients included exercise intolerance, myopathy, encephalomyopathy, cardiomyopathy, diabetes mellitus, sensorineural hearing impairment and progressive external ophthalmoplegia. The patients consisted of sporadic cases or cases with similar symptoms in maternal relatives. The ethics committee of the University of Oulu has approved the study protocol and all patients gave a written informed consent for participation in the study.

Anonymous healthy blood donors $(N=480)$ were used as population controls. It was required that the donor and his or her mother were born in the same region, and that the donor did not report neurological ailments, diabetes or sensorineural hearing impairment in the family. MtDNA haplogroups have been determined in all of the 480 controls [14], the hypervariable segment I (HVSI) in the D-loop has been sequenced in 403 controls [15] and the entire mtDNA sequence has been determined in 192 controls [14]. The ethics committee of the Finnish Red Cross has approved the study protocol.

\section{Molecular methods}

Total DNA was extracted from blood by using the QIAmp Blood Kit (Qiagen, Hilden, Germany). Total
DNA from muscle samples was extracted by using Wiz$\operatorname{ard}^{\circ}$ Genomic DNA purification kit (Promega, Madison, WI, U.S.A.) and standard tissue DNA extraction method using phenol and chloroform. MtDNA haplogroups were determined by restriction fragment analysis [16].

Forty-one samples were analysed by using conformation sensitive gel electrophoresis (CSGE) as previously described $[17,18]$. The mtDNA coding region, nucleotides m.577-m.16090, was amplified in 63 partially overlapping fragments. Each amplified fragment was mixed with the corresponding fragment amplified on a control template with a known sequence. Heteroduplex formation was also allowed to occur autogenously enabling the detection of heteroplasmic mutations. Heteroduplexes that differed in mobility in CSGE were sequenced (ABI PRISM ${ }^{ } 377$ Sequencer using DYEnamic ET Terminator Cycle Sequencing Kit; Amersham Pharmacia Biotech Inc., Buckinghamshire, U.K.) after purification with exonuclease I and shrimp alkaline phosphatase [19]. The primers used for sequencing were the same as those used for the amplification of the 63 CSGE fragments. Twenty-five samples were sequenced directly for their entire mtDNA. The mtDNA was first amplified in 11 fragments followed by sequencing using primers that were used for the amplification of mtDNA fragments.

Muscle DNA samples $(N=30)$ were examined for multiple mtDNA deletions by using long-PCR (Phusion expand XL-PCR, Boehringer Mannheim, Mannheim, Germany) as described previously [20]. Certain POLG1 mutations are fairly common in the Finnish population and, hence, the entire POLG1 gene (NCBI Reference Sequence: NM_002693) was sequenced in the two samples that were found to harbour multiple mtDNA deletions. Next-generation sequencing would have provided an effective tool for mtDNA analysis and heteroplasmy detection but, unfortunately, this method was beyond our resources at the time of investigation.

\section{Analysis of mtDNA variants}

Sequences were compared to the revised Cambridge reference sequence [GenBank: NC_012920] [21] and to mtDNA sequences available in the Mitomap database http://www.mitomap.org [22]. Variants were considered rare if they occurred $\leq 10$ times in the Mitomap database among 32,059 mtDNA sequences. Novel substitutions were confirmed by RFLP or by sequencing in both directions at least twice from different PCR products. The possibility of m.15933G > A being an artefact caused by nuclear mitochondrial DNA was examined by amplifying total mtDNA of patient 41 with long-PCR (XL-PCR) Expand Long Template PCR system kit (Boehringer Mannheim) as described previously [20] and by subsequently sequencing the mutation site with a forward primer at m.15714 and a reverse primer at m.16090. 
Conservation of nucleotides among species was evaluated using Mamit tRNA: Compilation of Mammalian mitochondrial tRNA genes http://mamit-tRNA.u-stra sbg.fr [23] in the case of tRNA genes and GiiB-JST mtSAP evaluation http://mtsnp.tmig.or.jp/cgi-bin/mts $\mathrm{np} /$ specAlign/ctrlSpecAlignE.cgi in the case of structural mtDNA genes [24]. The pathogenicity of $\mathrm{m} .15933 \mathrm{G}>\mathrm{A}$ was assessed by means of a multifactorial probabilitybased classification method PON-mt-tRNA that predicts the pathogenicity of single nucleotide substitutions in human mitochondrial tRNAs [25].

\section{Analysis of nonsynonymous mtDNA mutations}

Nonsynonymous mtDNA mutations were analysed for their pathogenic potential by using PolyPhen- 2 version 2.2.2 [26] http://genetics.bwh.harvard.edu/pph2/, SIFT B Link [27] http://sift.jcvi.org/www/SIFT_BLink_submit. html, PMut [28, 29] http://mmb2.pcb.ub.es/PMut/ and SNAP [30] http://rostlab.org/services/snap/ algorithms. Pathogenicity classes were ascertained according to the guidelines suggested by Wallis et al. 2013 and Claustres et al. 2014 [31, 32].

PolyPhen-2 calculates a naïve Bayes posterior probability for each mutation to assess its damaging potential. Mutations are then classified as benign if the probability is less than $50 \%$, possibly damaging if the probability is greater than $50 \%$ and probably damaging if the probability is greater than $90 \%$.

SIFT BLink and PMut algorithms are based on sequence homology. These algorithms assume that functionally important protein sequences are conserved in evolution, whereas diverse positions are unimportant to protein function. SIFT scores for nonsynonymous mutations range between $0-1$ and scores of $\leq 0.05$ are considered to be deleterious. PMut also uses information on sequence conservation and neural networks to predict whether a variant is benign or pathogenic. PMut provides a reliability index ranging from 0 (most unreliable) to 9 (most reliable). SNAP predicts functional effect by using conservation and functional information of proteins, such as secondary structure and solvent accessibility. A variant is assigned either neutral or non-neutral. A reliability index ( 0 being the most reliable prediction and 7 being the most unreliable) and an accuracy percentage is also provided.

Exact test of population differentiation as implemented in Arlequin 3.5 was used to compare frequencies of mtDNA haplogroups between cases and controls [33]. Phylogenetic networks of mtDNA sequences were constructed on the basis of median algorithm [34].

\section{Results}

MtDNA sequence variation and novel mutations

The frequency of mtDNA haplogroups did not differ between the 66 patients with suspected mitochondrial disease and the controls. Three novel homoplasmic variants, m.8743G $>\mathrm{C}, \mathrm{m} .11322 \mathrm{~A}>\mathrm{G}$ and $\mathrm{m} .15933 \mathrm{G}>\mathrm{A}$ were discovered (Fig. 1). The m.11322A $>\mathrm{G}$ variant (p. N188S, MTND4) was found in two siblings, one (patient 21) with hypertrophic cardiomyopathy, chronic atrial fibrillation and sick sinus syndrome and the other (patient 22) with ptosis, restricted eye movements and exercise intolerance. The nucleotide position $\mathrm{m} .11322$ is not notably conserved among 60 species which were investigated with GiiB-JST mtSAP evaluation http://mtsnp.tmig.or.jp/cgi-bin/mtsnp/specAlign/ctrlSpecAlignE.cgi [24]. The other two novel variants $\mathrm{m} .8743 \mathrm{G}>\mathrm{C}$ (p.V73L, MTATP6) and m.15933G $>\mathrm{A}$ in MTTT were found in the muscle and blood of patient 41 . The nucleotide at position $\mathrm{m} .8743$ is not particularly conserved, whereas m.15933G is a highly conserved (Table 1, Fig. 2). The m.15933G > A mutation was predicted to be pathogenic with a probability score of 0.92 by PON-mt-tRNA method. Sequencing revealed that the m.15933G $>$ A variant was present in an amplicon obtained in a longrange PCR amplification covering the whole mtDNA of patient 41.

Patient 41 is a 51-year old woman. Her athletic achievements did not match those of the other girls at school. In adulthood she has had poor muscle fitness, and exerciseinduced muscle weakness and myalgia. In addition, she has mild dysphagia and choking tendency. In her forties a nonsymmetrical ptosis developed and her right eye was operated at age 47 years. Psoriatic skin rash developed at age 49 years. Clinical neurological examination and blood lactate were normal. Muscle histology revealed ragged red fibers (RRF) and COX-negative fibers.

In addition, three other variants were considered rare occurring $\leq 10$ times in the Mitomap database of 32,059 sequences. The rare insertion m.7585_7586insT $(N=4$ in Mitomap database) between MTTD and MTCO2 was found in patient 16. This insertion adds a thymidine between the last nucleotide of MTTD and the first nucleotide of MTCO2 (Fig. 2). Cloning revealed that the insertion was present in all 100 clones investigated suggesting that the mutation in muscle mtDNA is homoplasmic with high probability. The other rare variants were m.4924G $>\mathrm{C}$ (p. S152T, MTND2) and m.7706G > A (p. A41T, MTCO2). The frequency of the rare nonsynonymous variants among the 66 patients did not differ from that in 89 haplogroup-matched controls drawn from the population [14].

\section{In-silico analysis of nonsynonymous variants}

All the nonsynonymous mtDNA variants $(N=47)$ discovered in the 66 patients were analysed for their pathogenic potential using PolyPhen-2, SIFT BLink, PMut and SNAP algorithms. Five of the mutations were predicted to be deleterious by at least three of the algorithms 


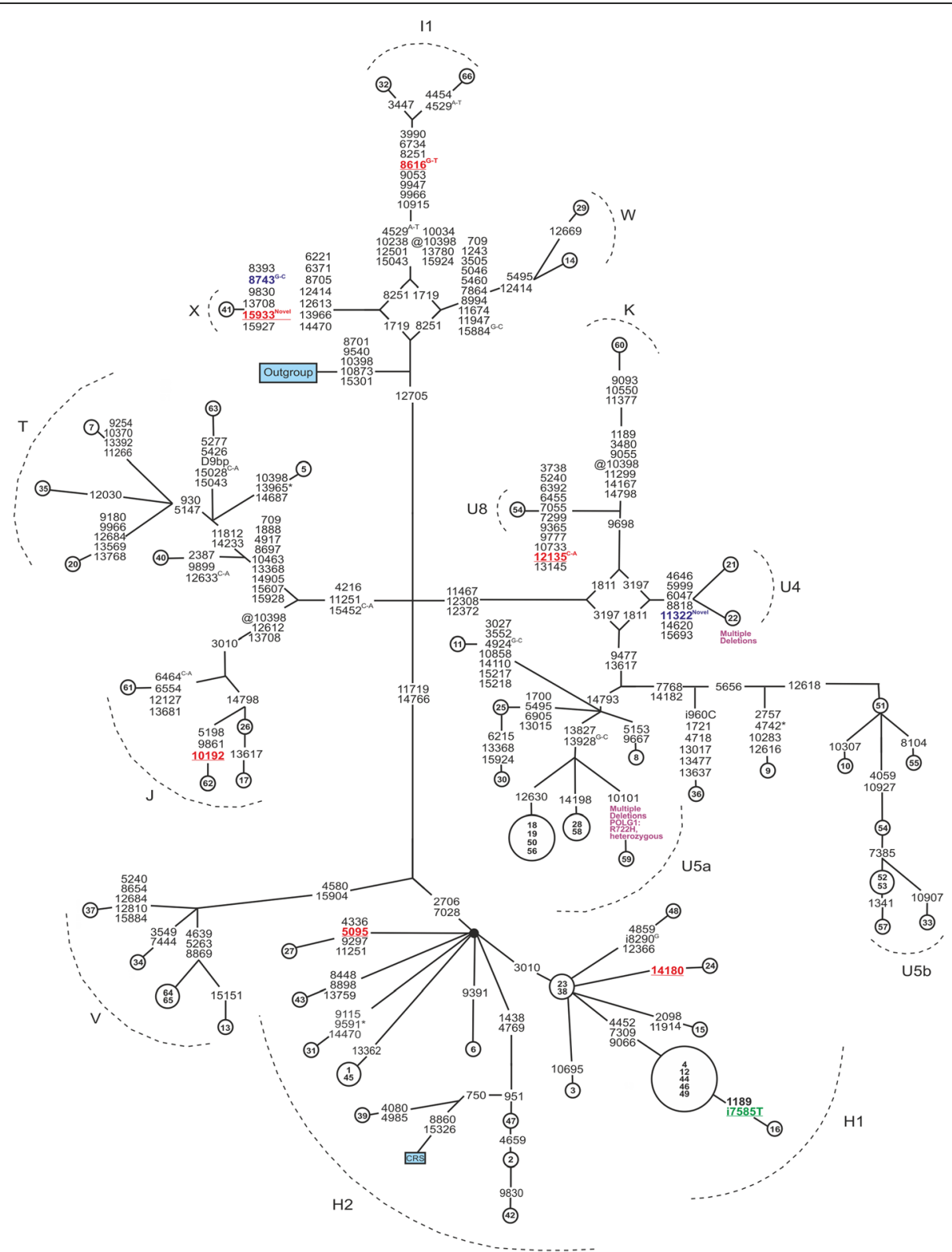

Fig. 1 Phylogenetic network of the mtDNA coding region in 66 patients with suspected mitochondrial disease. Patients are identified by numbers inside the nodes. Outgroup = an African sequence (GenBank: AF346980); CRS = the revised Cambridge Reference Sequence (GenBank: NC_012920). Unless marked otherwise, mtDNA variants are transitions. Superscript text indicates transversions. Insertions are marked with superscript (i), back mutations (@) and heteroplasmic mutations are marked with an asterisk (*). Underlined red font, variants predicted to be likely pathogenic, blue font novel mutations, green font rare mutations, purple font multiple mtDNA deletions and POLG1 mutations

(Table 2). The highest probability in favour of a pathogenic effect was obtained for m.8616G $>\mathrm{T}$ (p. L30F, MTATP6). With the exception of m.5095 T > C (p.I209T, MTND2) and m.14180 T > C (p. Y165C, MTND6), the three other variants predicted to be deleterious have previously been reported to be haplogroup-associated.

\section{Multiple mtDNA deletions}

Muscle samples from 30 patients were analysed for multiple mtDNA deletions. Patients 59 and 22 (Fig. 1) were found to harbour multiple deletions. Patient 59 suffered from bilateral progressive external ophthalmoplegia, diplopia, ptosis and mild exercise intolerance. His muscle histology revealed numerous RRFs and COX-negative muscle fibers. He harboured a heterozygous p.R722H allele in POLG1, but no potentially pathogenic mtDNA mutations.

Patient 22 harboured multiple mtDNA deletions and the novel m.11322A > G variant (p. N188S, MTND4), but not POLG1 mutations. Her daughter had increased 
Table 1 Evolutionary conservation analysis of the novel m.15933G > A mutation in MTTT among mammals

\begin{tabular}{|c|c|c|}
\hline & T-stem & T-loop \\
\hline Patient 41 & $\begin{array}{l}{ }^{49} \mathbf{C} \mathrm{C} T \mathrm{~T} \\
{ }^{65} \overline{\overline{\mathbf{A}}} \mathrm{A} A \mathrm{~A} A\end{array}$ & $\mathrm{ACCT}$ \\
\hline Homo sapiens & $\begin{array}{l}{ }^{49} \mathrm{C} T \mathrm{~T} \\
{ }^{65} \bar{G} A A A\end{array}$ & $\mathrm{ACCT}$ \\
\hline Pan paniscus & $\begin{array}{l}{ }^{49} \mathrm{C} T \mathrm{~A} A \\
{ }^{65} \overline{\mathrm{G}} \mathrm{AAA}\end{array}$ & $\mathrm{ACTT}$ \\
\hline Pan trogdolytes & $\begin{array}{l}{ }^{49} \mathrm{CTTC} \\
{ }^{65} \bar{G} \mathrm{GAA}\end{array}$ & $\mathrm{ACTT}$ \\
\hline Gorilla gorilla & $\begin{array}{l}{ }^{49} \mathrm{C} T \mathrm{TC} \\
{ }^{65} \overline{\mathrm{G}} \mathrm{AAG}\end{array}$ & ACCTC \\
\hline Hylobates lar & $\begin{array}{l}{ }^{49} \mathrm{CTTC} \\
{ }^{65} \overline{\mathrm{G}} \mathrm{AAA}\end{array}$ & TCTTC \\
\hline Rattus norwegicus & $\begin{array}{l}{ }^{49} \mathrm{C} T \mathrm{C} \\
{ }^{65} \overline{\mathrm{G}} \mathrm{AAG}\end{array}$ & AGTCT \\
\hline Mus musculus & $\begin{array}{l}{ }^{49} \mathrm{C} T \mathrm{TC} \\
{ }^{65} \overline{\mathrm{G}} \mathrm{A} A \mathrm{G}\end{array}$ & ATCTT \\
\hline Bos taurus & $\begin{array}{l}{ }^{49} \mathrm{C} C T C \\
{ }^{65} \overline{\mathrm{G}} \mathrm{GAG}\end{array}$ & AACAC \\
\hline
\end{tabular}

Mammalian MTTT sequences covering the T-stem base pairs and the T-loop. The nucleotide position m.15933 in consensus position 49 and its pair in position 65 are shown in the superscripts and the nucleotides are underlined

lactate in blood. No mtDNA deletions were detected in her brother (patient 21).

\section{Discussion}

We discovered three previously unreported mtDNA variants, three rare variants and a total of 47 nonsynonymous variants among 66 patients with suspected mitochondrial disease. In addition, two patients harbored multiple
mtDNA deletions. One of the novel variants was m.15933G > A in MTTT in a patient with ptosis, mild muscle weakness, myalgia, mild dysphagia, psoriasis and with RRF and COX-negative fibers in skeletal muscle. This variant is situated at the base of the T stem of $\mathrm{tRNA}^{\mathrm{Thr}}$ in tRNA consensus position 49, which is highly conserved among mammals, including its pair nucleotide at position 65 [35]. Nuclear mitochondrial DNA as a source of m.15933G > A was excluded by whole mitochondrial genome amplification and subsequent sequencing. The insertion m.15933_15934insG in the same position has been reported in one subject (GenBank: JQ702629.1) [36]. This insertion is not considered to be deleterious, because it makes the preceding variable loop one base pair longer but it retains the conserved m.15933G. On the other hand, the m.15933G > A removes the highly conserved G in the beginning of the $T$ stem and decreases the length of the $\mathrm{T}$ stem to four base pairs (Fig. 2). Another example of a similar pair of a pathogenic mutation and a polymorphic insertion is present in the $\mathrm{T}$ stem of MTTL2, where m.12311 $\mathrm{T}>\mathrm{C}$ is pathogenic and m.12311_12312insA is polymorphic variant $[35,37,38]$. Altogether six confirmed pathogenic mutations have been reported in MTTT [22, 23]. The symptoms of the patient, the conserved nature of the nucleotide site and the predicted high probability in favour of pathogenicity suggest that m.15933G $>A$ is pathogenic, but further functional studies will be needed to discern the biochemical consequences of the mutation.

The patient with the novel m.15933G $>$ A harboured also the novel variant m.8743G > C (p.V73L, MTATP6). This position holds a known polymorphism of m.8743G > A (p.V73M, MTATP6), but the transversion

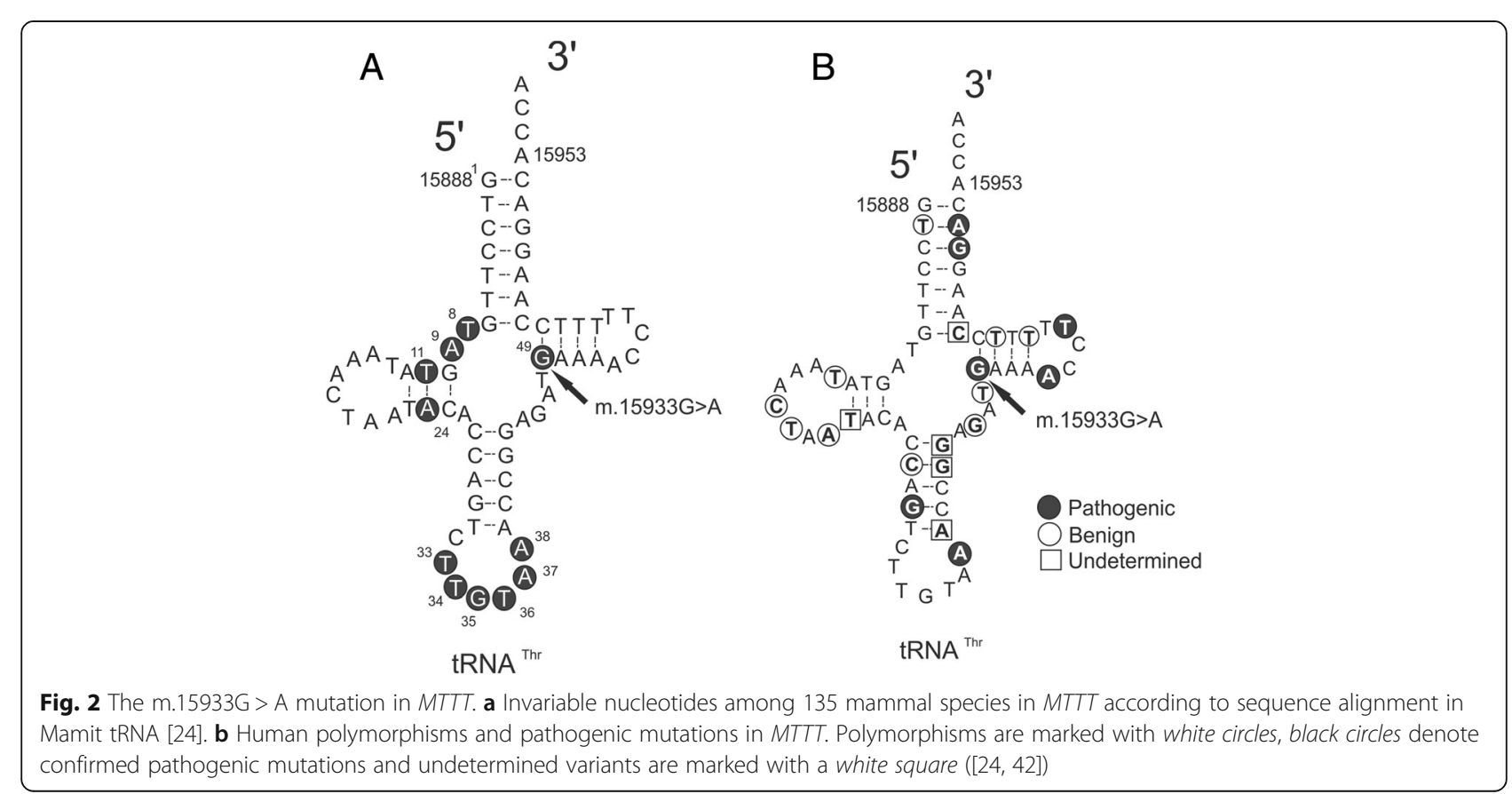


Table 2 Nonsynonymous mtDNA variants predicted to be likely pathogenic

\begin{tabular}{|c|c|c|c|c|c|c|c|c|}
\hline Variant & Protein change & Gene & PolyPhen-2 (\%) & SIFT Blink ${ }^{b}$ & SNAPC & Pmut $^{d}$ & Haplogroup association & Patient haplogroup \\
\hline m.5095 T >C & p.I209T & MTND2 & 99.8 & 0 & Pat/58\% & Pat/7 & Multiple & $\mathrm{H} 2$ \\
\hline $\mathrm{m} .8616 \mathrm{G}>\mathrm{T}$ & p.L30F & MTATP6 & 99.9 & 0 & Neutral/85\% & Pat/7 & I1, M74 & 11 \\
\hline m.10192C > T & p.S45F & MTND3 & 0 & 0.02 & Pat/58\% & Pat/6 & J1 & J1 \\
\hline m.12135C > A & p.S459Y & MTND4 & 90.6 & 0 & Pat/63\% & Pat/9 & U8, B4 & U8 \\
\hline m.14180 T > C & p.Y165C & MTND6 & 99.7 & 0.02 & Pat/63\% & Pat/7 & $\mathrm{L} 2, \mathrm{~T} 2, \mathrm{D} 4$ & $\mathrm{H} 1$ \\
\hline
\end{tabular}

${ }^{\text {a }}$ olyPhen-2 estimated probability of the variant being damaging: probabilities $\sim 50 \%$ are classified as possibly damaging and probabilities $>90 \%$ are classified as probably damaging

${ }^{b}$ SIFT score ranges from 0 to 1 . The amino acid substitution is predicted damaging if the score is $\leq 0.05$, and tolerated if the score is $>0.05$

'SNAP assigns variants as neutral or non-neutral. An expected accuracy percent is given ranging from 50 to $100 \%$, lower than $50 \%$ accurate predictions are not reported

${ }^{\mathrm{d}}$ PMut predicts a variant either pathogenic or neutral. A reliability index is given, ranging from 0 (most unreliable) to 9 (most reliable)

The algorithms used for prediction included PolyPhen-2, SNAP, SIFT BLink and PMut. Variants were considered to be likely pathogenic if at least three algorithms predicted a damaging effect

m.8743G > C has not been previously reported in databases. This nucleotide position is variable and the amino acid at this position is not evolutionarily conserved. We consider this novel variant unlikely to be pathogenic.

The three rare variants included the insertion m.7585_7586insT that was discovered in a patient with a multi-organ phenotype. The m.7585_7586insT variant is interesting, as it adds a nucleotide in between the contiguous MTTD and MTCO2 genes and may interfere with translation by one of three mechanisms. First, the MTTD transcript could be one nucleotide longer thus interrupting its proper function. Alternatively, the m.7585_7586insT insertion may affect the translation of $M T C O 2$. The third possible mechanism entails that the RNAase cleavage site in between MTTD and MTCO2 is disrupted. However, m.7585_7586insT has previously been discovered in four subjects belonging to haplotype H35, three of whom are Finnish. Our patient belonged to haplotype $\mathrm{H} 1$ suggesting that m.7585_7586insT has arisen at least twice in this population. Additional studies are needed to verify, if m.7585_7586ins T indeed affects the function of mitochondria. Unfortunately, only a few blood samples were available from this family and no autopsy had been performed on the proband after his death.

The frequency of rare nonsynonymous variants among the 66 patients did not differ from that in 89 haplogroup-matched controls drawn from the population [14]. Five out of the 47 nonsynonymous variants were predicted to be pathogenic in effect. Four algorithms were used including SNAP, SIFT BLink, PolyPhen-2 and PMut. These algorithms have been reported to accurately predict $76 \%$ of non-neutral variants, and SNAP is among the most accurate methods for predicting damaging variants [39]. We assumed the best candidates for pathogenic mutations to be those that three out of four algorithms predicted to be pathogenic. Three out of five predicted pathogenic variants were haplogroup-associated and, indeed, we found these variants in the same haplogroups as previously reported, suggesting that they are haplogroup-associated polymorphisms. On the other hand, m.5095 T > C (p.I209T, MTND2) has previously been reported in multiple haplogroups (B, C, T, A, U5a, R) [23], whereas we discovered it in haplogroup $\mathrm{H}$. This patient presented with proximal muscle weakness and atrophy, orbicularis oculi muscle weakness, myalgia and a large psoriasis-type rash. Inflammatory changes were detected in muscle histology. The true pathogenicity of $\mathrm{m} .5095 \mathrm{~T}>\mathrm{C}$ remains, however, unknown despite the high pathogenicity prediction score, as this mutation has been discovered in healthy individuals. Multiple mtDNA deletions were discovered in two patients aged 33 and 42 years with suspected mitochondrial disease. Multiple mtDNA deletions do occur normally in the course of healthy aging, but up to $48 \%$ of younger mitochondrial disease patients with multiple deletions harbour POLG1 mutations [40]. We found a heterozygous p.R722H allele in one patient, while the other patient with multiple deletions did not harbour any POLG1 mutations. The clinical features of the patient with the heterozygous p.R722H allele included progressive external ophthalmoplegia, diplopia, ptosis, mild exercise intolerance, and numerous RRFs and COX negative fibers in skeletal muscle. The p.R722H POLG1 allele has not been associated with disease in a heterozygous state, whereas in the homozygous state it leads to progressive external ophthalmoplegia, sensorineural hearing impairment, diabetes mellitus, dysphagia, a limb myopathy and dementia [41]. Patient 22 with multiple deletions, but without POLG1 mutations, also harboured a novel nonsynonymous mtDNA mutation m.11322A $>$ G (p. N188S, MTND4) that was predicted to be neutral.

\section{Conclusions}

We determined the coding region mtDNA sequence of 66 Finnish patients with suspected mitochondrial disease. Among the 47 nonsynonymous variants, five were predicted to be deleterious in effect by using four 
algorithms in parallel, but three of them were considered haplogroup-associated polymorphisms. A likely pathogenic novel mutation m.15933G > A (MTTT) was discovered in patient with a suspected mitochondrial disease. Sequencing of the mtDNA coding region is recommended for patients presenting with symptoms of a mitochondrial disorder but lacking the common disease-causing mutations in mtDNA.

\section{Abbreviations}

COX: Cytochrome oxidase; CRS: The Cambridge reference sequence; CSGE: Conformation sensitive gel electrophoresis; D-loop: Displacement loop, non-coding mtDNA segment; HVSI: Hypervariable segment l; MELAS: Mitochondrial encephalomyopathy, lactic acidosis with stroke-like episodes; MtDNA: Mitochondrial DNA; MTTT: Mitochondrial tRNA Threonine gene; NARP: Neuropathy, ataxia and retinitis pigmentosa; nDNA: Nuclear DNA; POLG1: Polymerase $\gamma$ gene; RFLP: Restriction fragment length polymorphism; RRF: Ragged red fiber

\section{Acknowledgements}

We greatly appreciate the technical assistance of Ms. Anja Heikkinen and Ms. Pirjo Keränen.

\section{Funding}

This study was supported by grants from Academy of Finland (project numbers 107174, 127764 and 266498), the Sigrid Juselius Foundation, the Finnish Graduate School for Population Genetics, the Päivikki and Sakari Sohlberg Foundation, The Alma and K.A. Snellman Foundation and the University of Oulu, Finland.

\section{Availability of data and materials}

Patient mtDNA sequences are available in GenBank: KY496857-KY496890.

\section{Authors' contributions}

$\mathrm{RH}, \mathrm{JU}$ and KM designed the study. HKS, AV, LK and RH generated and analysed the data. MK and JSM collected and analysed patient data. HKS and KM wrote the paper with contributions from other authors. All authors read and approved the final manuscript.

\section{Competing interests}

The authors declare that they have no competing interests.

\section{Consent for publication}

Not applicable.

\section{Ethics approval and consent to participate}

The ethics committee of the University of Oulu has approved the study protocol. All procedures performed in studies involving human participants were in accordance with the ethical standards with the 1964 Helsinki declaration and its later amendments. Informed consent was obtained from all individual participants included in the study.

\section{Author details}

${ }^{1}$ Research Unit of Clinical Neuroscience, Neurology, University of Oulu, P.O. Box 5000FI-90014 Oulu, Finland. ${ }^{2}$ Medical Research Center Oulu, Oulu University Hospital and University of Oulu, P.O. Box 5000FI-90014 Oulu, Finland. ${ }^{3}$ Department of Neurology, Oulu University Hospital, P.O. Box 20FI-90029 OYS Oulu, Finland. ${ }^{4}$ PEDEGO Research Unit, Pediatrics, University of Oulu, P.O. Box 5000FI-90014 Oulu, Finland. ${ }^{5}$ Department of Pediatrics, Oulu University Hospital, P.O. Box 23Fl-90029 OYS Oulu, Finland. ${ }^{6}$ Department of Clinical Genetics, Oulu University Hospital, P.O. Box 23FI-90029 OYS Oulu, Finland. ${ }^{7}$ PEDEGO Research Unit, Clinical Genetics, University of Oulu, P.O. Box 5000FI-90014 Oulu, Finland.
Received: 25 January 2016 Accepted: 3 February 2017

Published online: 10 February 2017

\section{References}

1. Chinnery PF, Elliott HR, Hudson G, Samuels DC, Relton CL. Epigenetics, epidemiology and mitochondrial DNA diseases. Int J Epidemiol. 2012:41(1):177-87.

2. Gorman GS, Schaefer AM, Ng Y, Gomez N, Blakely EL, Alston CL, Feeney C, Horvath R, Yu-Wai-Man P, Chinnery PF, Taylor RW, Turnbull DM, Mcfarland R. Prevalence of nuclear and mitochondrial DNA mutations related to adult mitochondrial disease. Ann Neurol. 2015:77(5):753-9.

3. Ylikallio E, Suomalainen A. Mechanisms of mitochondrial diseases. Ann Med. 2012:44(1):41-59.

4. Breen MS, Kondrashov FA. Mitochondrial pathogenic mutations are population-specific. Biol Direct. 2010;5:68.

5. Zhou X, Qian Y, Zhang J, Tong Y, Jiang P, Liang M, Dai X, Zhou H, Zhao F, Ji Y, Mo JQ, Qu J, Guan MX. Leber's hereditary optic neuropathy is associated with the T3866C mutation in mitochondrial ND1 gene in three Han Chinese families. Invest Ophthalmol Vis Sci. 2012;53(8):4586-94.

6. Cai W, Fu Q, Zhou X, Qu J, Tong Y, Guan MX. Mitochondrial variants may influence the phenotypic manifestation of Leber's hereditary optic neuropathy-associated ND4 G11778A mutation. J Genet Genomics. 2008; 35(11):649-55.

7. Shu L, Zhang YM, Huang XX, Chen CY, Zhang XN. Complete mitochondrial DNA sequence analysis in two southern Chinese pedigrees with Leber hereditary optic neuropathy revealed secondary mutations along with the primary mutation. Int J Ophthalmol. 2012;5(1):28-31.

8. Achilli A, Olivieri A, Pala M, Hooshiar Kashani B, Carossa V, Perego UA, Gandini F, Santoro A, Battaglia V, Grugni V, Lancioni H, Sirolla C, Bonfigli AR, Cormio A, Boemi M, Testa I, Semino O, Ceriello A, Spazzafumo L, Gadaleta MN, Marra M, Testa R, Franceschi C, Torroni A. Mitochondrial DNA backgrounds might modulate diabetes complications rather than T2DM as a whole. Plos One. 2011:6(6):e21029.

9. Soini HK, Moilanen JS, Finnila S, Majamaa K. Mitochondrial DNA sequence variation in Finnish patients with matrilineal diabetes mellitus. BMC Res Notes. 2012;5(1):350

10. Santoro A, Balbi V, Balducci E, Pirazzini C, Rosini F, Tavano F, Achilli A, Siviero P, Minicuci N, Bellavista E, Mishto M, Salvioli S, Marchegiani F, Cardelli M, Olivieri F, Nacmias B, Chiamenti AM, Benussi L, Ghidoni R, Rose G, Gabelli C, Binetti G, Sorbi S, Crepaldi G, Passarino G, Torroni A, Franceschi C. Evidence for sub-haplogroup h5 of mitochondrial DNA as a risk factor for late onset Alzheimer's disease. Plos One. 2010;5(8):e12037.

11. Coskun P, Wyrembak J, Schriner SE, Chen HW, Marciniack C, Laferla F, Wallace DC. A mitochondrial etiology of Alzheimer and Parkinson disease. Biochim Biophys Acta. 2012;1820(5):553-64.

12. Federico A, Cardaioli E, Da Pozzo P. Formichi P, Gallus GN, Radi E. Mitochondria, oxidative stress and neurodegeneration. J Neurol Sci. 2012:322(1-2):254-62.

13. Khusnutdinova E, Gilyazova I, Ruiz-Pesini E, Derbeneva O, Khusainova R, Khidiyatova I, Magzhanov R, Wallace DC. A mitochondrial etiology of neurodegenerative diseases: evidence from Parkinson's disease. Ann N Y Acad Sci. 2008:1147:1-20.

14. Finnilä S, Lehtonen MS, Majamaa K. Phylogenetic network for European mtDNA. Am J Hum Genet. 2001;68(6):1475-84.

15. Meinilä M, Finnilä $\mathrm{S}$, Majamaa K. Evidence for mtDNA admixture between the Finns and the Saami. Hum Hered. 2001:52(3):160-70.

16. Torroni A, Huoponen K, Francalacci P, Petrozzi M, Morelli L, Scozzari R, Obinu D, Savontaus ML, Wallace DC. Classification of European mtDNAs from an analysis of three European populations. Genetics. 1996;144(4):1835-50

17. Körkkö J, Annunen S, Pihlajamaa T, Prockop DJ, Ala-Kokko L. Conformation sensitive gel electrophoresis for simple and accurate detection of mutations: comparison with denaturing gradient gel electrophoresis and nucleotide sequencing. Proc Natl Acad Sci U S A. 1998;95(4):1681-5.

18. Finnilä S, Hassinen IE, Ala-Kokko L, Majamaa K. Phylogenetic network of the mtDNA haplogroup U in Northern Finland based on sequence analysis of the complete coding region by conformation-sensitive gel electrophoresis. Am J Hum Genet. 2000:66(3):1017-26.

19. Werle E, Schneider C, Renner M, Volker M, Fiehn W. Convenient single-step, one tube purification of $\mathrm{PCR}$ products for direct sequencing. Nucleic Acids Res. 1994;22(20):4354-5. 
20. Remes AM, Majamaa-Voltti K, Kärppä M, Moilanen JS, Uimonen S, Helander H, Rusanen H, Salmela PI, Sorri M, Hassinen IE, Majamaa K. Prevalence of large-scale mitochondrial DNA deletions in an adult Finnish population. Neurology. 2005;64(6):976-81.

21. Andrews RM, Kubacka I, Chinnery PF, Lightowlers RN, Turnbull DM, Howell $\mathrm{N}$. Reanalysis and revision of the Cambridge reference sequence for human mitochondrial DNA. Nat Genet. 1999;23(2):147.

22. Lott MT, Leipzig JN, Derbeneva O, Xie HM, Chalkia D, Sarmady M, Procaccio V, Wallace DC. mtDNA Variation and Analysis Using MITOMAP and MITOMASTER. Curr Protoc Bioinformatics. 2013;1(23):1-26.

23. Putz J, Dupuis B, Sissler M, Florentz C. Mamit-tRNA, a database of mammalian mitochondrial tRNA primary and secondary structures. RNA. 2007;13(8):1184-90.

24. Tanaka M, Cabrera VM, Gonzalez AM, Larruga JM, Takeyasu T, Fuku N, Guo $\sqcup$, Hirose R, Fujita Y, Kurata M, Shinoda K, Umetsu K, Yamada Y, Oshida Y, Sato Y, Hattori N, Mizuno Y, Arai Y, Hirose N, Ohta S, Ogawa O, Tanaka Y, Kawamori R, Shamoto-Nagai M, Maruyama W, Shimokata H, Suzuki R, Shimodaira H. Mitochondrial genome variation in eastern Asia and the peopling of Japan. Genome Res. 2004;14(10A):1832-50.

25. Niroula A, Vihinen M. PON-mt-tRNA: a multifactorial probability-based method for classification of mitochondrial tRNA variations. Nucleic Acids Res. 2016;44(5):2020-7

26. Adzhubei IA, Schmidt S, Peshkin L, Ramensky VE, Gerasimova A, Bork P, Kondrashov AS, Sunyaev SR. A method and server for predicting damaging missense mutations. Nat Methods. 2010;7(4):248-9.

27. Ng PC, Henikoff S. Predicting deleterious amino acid substitutions. Genome Res. 2001;11(5):863-74.

28. Ferrer-Costa C, Orozco M, de la Cruz X. Sequence-based prediction of pathological mutations. Proteins. 2004;57(4):811-9.

29. Ferrer-Costa C, Gelpi JL, Zamakola L, Parraga I, de la Cruz X, Orozco M. PMUT: a web-based tool for the annotation of pathological mutations on proteins. Bioinformatics. 2005;21(14):3176-8.

30. Bromberg $Y$, Yachdav G, Rost B. SNAP predicts effect of mutations on protein function. Bioinformatics. 2008;24(20):2397-8.

31. Claustres M, Kozich V, Dequeker E, Fowler B, Hehir-Kwa JY, Miller K, Oosterwijk C, Peterlin B, van Ravenswaaij-Arts C, Zimmermann U, Zuffardi O, Hastings RJ, Barton DE, European Society of Human Genetics. Recommendations for reporting results of diagnostic genetic testing (biochemical, cytogenetic and molecular Genetic). Eur J Hum Genet. 2014;22(2):160-70.

32. Wallis Y, Payne S, Mcanulty C, Bodmer D, Sistermans E, Robertson K, Moore D, Abbs S, Deans Z, Devereau A. Practice guidelines for the evaluation of pathogenicity and the reporting of sequence variants in clinical molecular genetics. Birmingham: Association for Clinical Genetic Science (ACGS); 2013. p. 1-16.

33. Excoffier L, Laval G, Schneider S. Arlequin (version 3.0): an integrated software package for population genetics data analysis. Evol Bioinformatics Online. 2007;1:47-50.

34. Bandelt HJ, Forster $P$, Rohl A. Median-joining networks for inferring intraspecific phylogenies. Mol Biol Evol. 1999;16(1):37-48.

35. Vilmi T, Moilanen JS, Finnilä S, Majamaa K. Sequence variation in the tRNA genes of human mitochondrial DNA. J Mol Evol. 2005;60(5):587-97.

36. Behar DM, van Oven M, Rosset S, Metspalu M, Loogvali EL, Silva NM, Kivisild T, Torroni A, Villems R. A "Copernican" reassessment of the human mitochondrial DNA tree from its root. Am J Hum Genet. 2012;90(4):675-84.

37. Herrnstadt C, Elson JL, Fahy E, Preston G, Turnbull DM, Anderson C, Ghosh SS, Olefsky JM, Beal MF, Davis RE, Howell N. Reduced-median-network analysis of complete mitochondrial DNA coding-region sequences for the major African, Asian, and European haplogroups. Am J Hum Genet. 2002;70(5):1152-71.

38. van Oven M, Kayser M. Updated comprehensive phylogenetic tree of global human mitochondrial DNA variation. Hum Mutat. 2009;30(2):E386-94.

39. da Hao C, Feng Y, Xiao R, Xiao PG. Non-neutral nonsynonymous single nucleotide polymorphisms in human $A B C$ transporters: the first comparison of six prediction methods. Pharmacol Rep. 2011;63(4):924-34

40. Ferreira M, Evangelista T, Almeida LS, Martins J, Macario MC, Martins E, Moleirinho A, Azevedo L, Vilarinho L, Santorelli FM. Relative frequency of known causes of multiple mtDNA deletions: two novel POLG mutations. Neuromuscul Disord. 2011;21(7):483-8.
41. Komulainen $T$, Hinttala R, Kärppä M, Pajunen L, Finnilä S, Tuominen H, Rantala H, Hassinen I, Majamaa K, Uusimaa J. POLG1 p.R722H mutation associated with multiple mtDNA deletions and a neurological phenotype. BMC Neurol. 2010;10:29.

42. Ruiz-Pesini E, Lott MT, Procaccio V, Poole JC, Brandon MC, Mishmar D, Yi C, Kreuziger J, Baldi P, Wallace DC. An enhanced MITOMAP with a global mtDNA mutational phylogeny. Nucleic Acids Res. 2007;35(Database issue): D823-8.

\section{Submit your next manuscript to BioMed Central and we will help you at every step:}

- We accept pre-submission inquiries

- Our selector tool helps you to find the most relevant journal

- We provide round the clock customer support

- Convenient online submission

- Thorough peer review

- Inclusion in PubMed and all major indexing services

- Maximum visibility for your research

Submit your manuscript at www.biomedcentral.com/submit
Biomed Central 